\title{
Low birthweight is associated with an increased risk of LADA and type 2 diabetes: results from a Swedish case-control study
}

\author{
Rebecka Hjort $^{1}$ - Lars Alfredsson ${ }^{2}$ Per-Ola Carlsson ${ }^{3}$ - Leif Groop ${ }^{4} \cdot$ Mats Martinell $^{5}$. \\ Petter Storm ${ }^{4}$ - Tiinamaija Tuomi ${ }^{6,7,8} \cdot$ Sofia Carlsson ${ }^{1}$
}

Received: 5 May 2015 / Accepted: 6 July 2015 / Published online: 25 July 2015

(C) Springer-Verlag Berlin Heidelberg 2015

\begin{abstract}
Aims/hypothesis Our aim was to investigate the association between birthweight and latent autoimmune diabetes in adults (LADA), a common diabetes form with features of both type 1 and type 2 diabetes.

Methods We used data from the Epidemiological Study of Risk Factors for LADA and Type 2 Diabetes (ESTRID), a Swedish population-based study. Eligible for the analysis were 134 incident LADA cases (glutamic acid decarboxylase antibody [GADA] positive), 350 incident type 2 diabetes cases (GADA negative) and 603 randomly selected controls.
\end{abstract}

Electronic supplementary material The online version of this article (doi:10.1007/s00125-015-3711-8) contains peer-reviewed but unedited supplementary material, which is available to authorised users.

Rebecka Hjort

rebecka.hjort@ki.se

1 Unit of Epidemiology, Institute of Environmental Medicine, Karolinska Institutet, Box 210, 17177 Stockholm, Sweden

2 Unit of Cardiovascular Epidemiology, Institute of Environmental Medicine, Karolinska Institutet, Stockholm, Sweden

3 Department of Medical Sciences, Uppsala University, Uppsala, Sweden

4 Department of Clinical Sciences in Malmö, Clinical Research Centre, Lund University, Malmö, Sweden

5 Department of Public Health and Caring Sciences, Uppsala University, Uppsala, Sweden

6 Division of Endocrinology, Abdominal Center, Helsinki University Hospital, Helsinki, Finland

7 Finnish Institute for Molecular Medicine and Research Program for Diabetes and Obesity, University of Helsinki, Helsinki, Finland

8 Folkhalsan Research Center, Helsinki, Finland
We present ORs and 95\% CIs for LADA and type 2 diabetes in relation to birthweight, adjusted for sex, age, BMI and family history of diabetes.

Results Low birthweight increased the risk of LADA as well as the risk of type 2 diabetes; OR per $\mathrm{kg}$ reduction was estimated as $1.52(95 \%$ CI $1.12,2.08)$ and 1.58 (1.23, 2.04), respectively. The OR for participants weighing $<3 \mathrm{~kg}$ compared with $\geq 4 \mathrm{~kg}$ at birth was estimated as $2.38(1.23,4.60)$ for LADA and $2.37(1.37,4.10)$ for type 2 diabetes. A combination of low birthweight $(<3 \mathrm{~kg})$ and current overweight $(\mathrm{BMI} \geq 25)$ further augmented the risk: LADA, OR $3.26(1.69,6.29)$; and type 2 diabetes, OR $39.93(19.27,82.71)$. Family history of diabetes had little impact on these estimates.

Conclusions/interpretation Our results suggest that low birthweight may be a risk factor for LADA of the same strength as for type 2 diabetes. These findings support LADA, despite its autoimmune component, having an aetiology that includes factors related to type 2 diabetes.

Keywords Birthweight · BMI · Case-control study · Interaction - LADA - Latent autoimmune diabetes in adults . Type 2 diabetes
Abbreviations
ANDIS All New Diabetics in Skåne
ANDiU All New Diabetics in Uppsala
ESTRID Epidemiological Study of Risk Factors for LADA and Type 2 diabetes
GADA Glutamic acid decarboxylase antibody
LADA Latent autoimmune diabetes in adults
RERI Relative excess risk due to interaction 


\section{Introduction}

Low birthweight is associated with an increased risk of type 2 diabetes [1]. It has been hypothesised that this reflects the effects of poor nutrition of the fetus leading to permanent changes in glucose metabolism [2]. The underlying mechanisms are not clear, but a link between low birthweight and insulin resistance is well documented $[3,4]$ and an association with low insulin secretion has been reported $[5,6]$. Genetic factors contributing to in utero growth retardation and adult diabetes risk have also been implicated [7], suggesting an inherited predisposition to insulin resistance [8]. Less is known about autoimmune diabetes in the context of birthweight; a small but significant link between high birthweight and type 1 diabetes has been documented, most probably reflecting maternal diabetes, affecting both the fetal development and future diabetes risk $[9,10]$.

Latent autoimmune diabetes in adults (LADA) is an autoimmune form of diabetes with adult onset and slower progression than type 1 diabetes [11]. It may be the second most prevalent type of diabetes in Europe, accounting for $10 \%$ of all diabetes in the adult population [12]. Described as a hybrid form of diabetes, LADA also has features of type 2 diabetes as patients tend to be insulin resistant and overweight [11]. The similarities with type 2 diabetes imply that low birthweight may increase the risk of LADA as a result of the same underlying aetiology. The risk of LADA may also, like type 1 diabetes, be related only to high birthweight, reflecting an excess risk associated with diabetes in the mother [13]. To date, no attempts have been made to elucidate the association between birthweight and LADA.

Our aim was to explore the risk of LADA and type 2 diabetes in relation to birthweight, using data from a Swedish population-based study of incident cases of diabetes.

\section{Methods}

Study population We used data from the Epidemiological Study of Risk Factors for LADA and Type 2 diabetes (ESTRID), a population-based case-control study with incident cases (www.ki.se/IMM/ESTRID). The details of ESTRID have been described previously [14]. In short, ESTRID was initiated in 2010, recruiting incident cases of diabetes through diabetes registries in the Swedish counties of Skåne (All New Diabetics in Skåne [ANDIS], http://andis. ludc.med.lu.se/) and Uppsala (All New Diabetics in Uppsala [ANDiU], www.andiu.se/). The aim of these registers is to characterise all incident cases of diabetes according to type, genetic factors and clinical features.

All individuals with incident LADA registered in Skåne (2010-) and Uppsala (2012-) were invited to take part in ESTRID, together with a random sample of people with type
2 diabetes (four per LADA case) and control participants free from diabetes (six per LADA case). The controls were randomly selected via the National Population Registry, which covers the whole population of Skåne and Uppsala ( $\sim 1.6$ million inhabitants). They had to be $\geq 35$ years of age (corresponding to the criteria used to define LADA) and were matched on geographical area (county) and date of participation, i.e. incidence density sampling [15]. All participants in ESTRID between 1 September 2010 and 1 July 2014 with self-reported information on birthweight were eligible for the analyses presented here.

Of the original sample, $44.6 \%$ (Skåne) and $45.3 \%$ (Uppsala) of participants could recall their birthweight information, including 134 patients with LADA, 350 patients with type 2 diabetes and 603 controls. Those who filled in the questionnaire but did not remember their birthweight (answered 'I do not know' to the birthweight question) were excluded from the analysis. The overall response rates for ESTRID were $80 \%$ for cases and $66 \%$ for controls. Participants gave written informed consent and the study was approved by the Ethical Review Board in Stockholm (reference number 2010/336-31/2).

Assessment of exposure and covariates A comprehensive questionnaire soliciting a broad range of items on lifestyle, family history and health was sent out by mail to all cases and their matched controls. Patients received the questionnaire close to diagnosis, with careful instructions to report lifestyle habits as they had been before diagnosis.

Birthweight Information on birthweight was based on selfreports. To validate the self-reported information on birthweight, ESTRID was linked to the Swedish Medical Birth Register. This registry contains information on prenatal and neonatal care for nearly all births in Sweden from 1973 onwards; information on birthweight was collected for all ESTRID participants born in 1973 onwards $(n=112)$. Of these individuals, 76 (68\%), including 16 cases and 60 controls, also had self-reported information on birthweight from the ESTRID questionnaire.

Covariates The BMI was calculated by self-reported weight and height as $\mathrm{kg} / \mathrm{m}^{2}$ and participants were categorised based on WHO criteria as normal weight $\left(\mathrm{BMI}<25 \mathrm{~kg} / \mathrm{m}^{2}\right)$, overweight $\left(\mathrm{BMI} \geq 25 \mathrm{~kg} / \mathrm{m}^{2}\right)$ and obese $\left(\mathrm{BMI} \geq 30 \mathrm{~kg} / \mathrm{m}^{2}\right)$. Detailed information on family history of diabetes was gathered through questions on diabetes in first-degree relatives (mother, father, sister, brother and children) and second-degree relatives (maternal and paternal grandparents, aunts and uncles). Relatives were classed as having type 1 diabetes if they had onset <age 40 and insulin treatment, but were otherwise deemed to have type 2 diabetes. Responders were also asked to report their highest level of education, with the categories 
elementary, high school and university. Smoking status was collected by questions on smoking habits and respondents were divided into current, former or never smokers. Alcohol intake was assessed through questions on amount and frequency of wine, beer and liquor intake, with participants classed as abstainers, ex-drinkers, or low, moderate or high consumers based on this information. Information on physical activity was obtained by questions on leisure time activity during the preceding year, with four response options ranging from sedentary to very active.

Clinical data and case definition Patients were diagnosed within the healthcare system of Skåne and Uppsala and registered in the ANDIS (Skåne) and ANDiU (Uppsala) registries. At the time of diagnosis, blood samples from all cases were collected and analysed for glutamic acid decarboxylase antibody (GADA), C-peptide and fasting blood glucose levels at the central laboratory of the university hospital of each county.

Positivity for GADA, which is the most common autoantibody, being present in $90 \%$ of all individuals with adult autoimmune diabetes [12], was measured by ELISA, with cut-off $\geq 10 \mathrm{U} / \mathrm{ml}$, according to the manufacturer's directions [16]. The values are reported as an index value in relation to standard serum. The assay gives a maximum value of 250 with a cut-off level $10.7 \mathrm{U} / \mathrm{ml}$; when GADA was measured in $\mathrm{Ca}^{2+}$ treated plasma, sensitivity and specificity were estimated as $84 \%$ and $98 \%$, respectively [16].

Assessment of C-peptide level was performed using a Cobas e 601 analyser, (Roche Diagnostics, Mannheim, Germany) or IMMULITE 2000 (Siemens Healthcare Diagnostics Products, Llanberis, UK) [17].

For this study, LADA was defined as onset $\geq 35$ years of age, GADA positivity $(\geq 10 \mathrm{U} / \mathrm{ml})$ and C-peptide levels $\geq 0.3 \mathrm{nmol} / \mathrm{l}$ (Cobas e 601) or $\geq 0.2 \mathrm{nmol} / 1$ (IMMULITE). Individuals with onset $\geq 35$ years but who were GADA negative $(<10 \mathrm{U} / \mathrm{ml})$ and with $\mathrm{C}$ peptide levels $>0.72 \mathrm{nmol} / \mathrm{l}$ (Cobas e 601) or $>0.6 \mathrm{nmol} / \mathrm{l}$ (IMMULITE) were classed as having type 2 diabetes. There is no universal definition of LADA, but this definition is in line with previous reports, except for C-peptide which was used as an indicator of remaining insulin production and a slow 'latent' onset. This replaced the commonly used insulin criterion, which is subjective. Via linkage to the ANDIS and ANDiU registries, clinical data from the time of diagnosis were available for all patients who gave consent for this $(98.8 \%$ of all cases; two LADA cases and four type 2 diabetes cases are missing in these analyses). The HOMA model was used to estimate insulin resistance (HOMA-IR) and beta cell function (HOMA- $\beta$ ) based on fasting plasma glucose and C-peptide [18]. Information from blood samples was not available for the controls in the present study.
Statistical analysis By means of logistic regression we calculated ORs with $95 \%$ CIs for the associations for birthweight, LADA and type 2 diabetes. Conditional logistic regression analysis, matched for date of participation and residential area, was also conducted, but we present only the results from the unmatched analyses as these were in close agreement with those from the matched analyses, but allowed us to use all the available information. The ORs were interpreted as incidence rate ratios, as this case-control study was based on incident cases and controls sampled by incidence density sampling [15].

The ORs were adjusted for age (continuous) and sex (model 1), age, sex and BMI (continuous) (model 2) and age, sex, BMI and family history of diabetes (yes vs no) (model 3). Results from model 3 are presented unless otherwise stated. Information on these covariates was complete for all participants with information on birthweight. Additional adjustment for education, smoking, alcohol and physical activity did not affect the ORs and therefore these variables were not included in the final model. Birthweight was modelled both as a continuous variable (per kg reduction in birthweight) and as a categorical variable divided into low $(<3 \mathrm{~kg})$, normal (3-<4 kg) and high $(\geq 4 \mathrm{~kg})$ birthweight. We also modelled birthweight in five categories with reference to the WHO definition of low birthweight $(<2.5 \mathrm{~kg}, 2.5-<3 \mathrm{~kg}, 3-<3.5 \mathrm{~kg}$, $3.5-<4 \mathrm{~kg}$ and $\geq 4.0 \mathrm{~kg}$ ) [19]. To determine possible interactions between low birthweight and overweight we calculated the relative excess risk due to interaction (RERI) [20]. In these analyses, birthweight and BMI were dichotomised as low $(<3 \mathrm{~kg})$ and high $(\geq 3 \mathrm{~kg})$ birthweight and normal weight $\left(\mathrm{BMI}<25 \mathrm{~kg} / \mathrm{m}^{2}\right)$ vs overweight/obesity $\left(\mathrm{BMI} \geq 25 \mathrm{~kg} / \mathrm{m}^{2}\right)$. Sensitivity analyses were performed using a higher cut-off for the GADA assay $(\geq 20 \mathrm{U} / \mathrm{ml}$ rather than $\geq 10 \mathrm{U} / \mathrm{ml})$. We also performed separate analyses in which: participants reporting diabetes in the mother were excluded to account for maternal diabetes during pregnancy; and participants born outside Sweden or with immigrant parents were excluded, to check if ethnicity could affect the results. Analyses were performed separately for individuals older than 60 years as we could not validate self-reported birthweight in higher age groups and participants' recall may have been less reliable.

Two-sided $p$ values were calculated using $\chi^{2}$ (proportion), Student's $t$ (mean) and Kruskal-Wallis (median) tests. All statistical calculations were performed using the statistical software program SAS version 9.4 (SAS Institute, Cary, NC, USA). Where ORs are reported, the 95\% CI are shown in parentheses.

\section{Results}

Validity of self-reported birthweight The correlation between self-reported and registry-based information on 
birthweight was $0.86(p<0.0001)$. For patients this estimate was $0.91(p<0.0001)$ and for controls $0.83(p<0.0001)$. The self-reporting method had a sensitivity of $90 \%$ and $100 \%$ specificity for participants with low $(<3 \mathrm{~kg})$ birthweight. All patients were correctly classed according to birthweight category $(100 \%$ sensitivity and $100 \%$ specificity), whereas one control with low birthweight was classed incorrectly in the normal weight group $(3-<4 \mathrm{~kg})$. With regard to nonresponders to the birthweight question, i.e. ESTRID participants not included in the present study population, we compared registry information on birthweight for those with and without self-reported information on birthweight and found that it was similar: $3.44 \mathrm{~kg}$ vs $3.45 \mathrm{~kg}$. If we compare register data for the controls with and without self-reported birthweight $(n=81)$, we find that those without self-reported weight have a slightly higher mean birthweight $(3.55 \mathrm{~kg}$ vs $3.43 \mathrm{~kg}$ ).

Characteristics Controls had the same mean age (54.7 years) as patients with LADA, but were leaner (Table 1) and had a substantially lower prevalence of family history of diabetes. Compared with patients with type 2 diabetes, LADA patients were leaner and younger, had lower levels of C-peptide, poorer beta cell function and were more often on insulin treatment $(58.0 \%$ vs $4.3 \%)$, whereas no difference in insulin resistance was detected. Family history of type 1 diabetes was more frequent in patients with LADA than in those with type 2 diabetes, whereas family history of type 2 diabetes tended to be more common in the latter group. The proportion with low $(<3 \mathrm{~kg})$ birthweight was higher in patients with LADA (21.6\%) and type 2 diabetes (22.3\%) than in controls (14.1\%).
LADA and birthweight The risk of LADA was inversely associated with birthweight (Table 2) and increased by $52 \%$ (OR 1.52 [95\% CI 1.12, 2.08]) with every kg reduction in birthweight. Compared with those with high $(\geq 4 \mathrm{~kg})$ birthweight, the OR for individuals with low $(<3 \mathrm{~kg})$ birthweight was estimated as $2.38(1.23,4.60)$. Exclusion of participants reporting maternal diabetes augmented this estimate slightly, OR 2.77 (1.33, 5.78). Sensitivity analysis using a higher cut-off level for the GADA assay $(20 \mathrm{U} / \mathrm{ml}$ instead of $10 \mathrm{U} / \mathrm{ml})$ indicated similar results, OR $1.49(1.07,2.06)$ per $\mathrm{kg}$ birthweight reduction. A finer division of birthweight into five strata indicated a graded trend, with the highest risk of LADA in those weighing less than $2.5 \mathrm{~kg}$ at birth, OR 2.90 (1.19, 7.06) compared with individuals weighing $\geq 4 \mathrm{~kg}$, but an elevated risk was still seen for those with a birthweight between 2.5 and $<3 \mathrm{~kg}$, OR $2.14(1.03,4.46)$. As shown in Table 2, adjustments for BMI and family history of diabetes had a minor influence on the risk estimates.

Type 2 diabetes and birthweight The risk of type 2 diabetes increased by $58 \%$ for every $\mathrm{kg}$ reduction in birthweight (OR $1.58[1.23,2.04])$ and the OR for low vs high birthweight was estimated as $2.37(1.37,4.10)$. A similar pattern was seen when dividing birthweight into five groups: ORs for those with the lowest weights $(<2.5 \mathrm{~kg}$ and $2.5-<3 \mathrm{~kg})$ were 2.48 $(1.16,5.28)$ and $2.32(1.23,4.37)$, respectively, compared with the highest weight group ( $\geq 4 \mathrm{~kg}$ ).

Interaction between low birthweight and adult overweight The excess risk of LADA was seen primarily in individuals with a combination of overweight and low birthweight $(<3 \mathrm{~kg})$

Table 1 Characteristics of the participants

\begin{tabular}{|c|c|c|c|c|}
\hline Characteristics & Controls & Type 2 diabetes & LADA & $p$ value \\
\hline No. of individuals & 603 & 350 & 134 & \\
\hline Men, $n(\%)$ & $246(40.8)$ & $185(52.9)$ & $65(48.5)$ & $0.4169^{\mathrm{a}}$ \\
\hline Age (years), mean (SD) & $54.7(12.3)$ & $59.5(9.2)$ & $54.7(11.1)$ & $<0.0001^{\mathrm{a}}$ \\
\hline BMI $\left(\mathrm{kg} / \mathrm{m}^{2}\right)$, mean $(\mathrm{SD})$ & $25.6(4.2)$ & $32.1(5.8)$ & $26.9(5.2)$ & $<0.0001^{\mathrm{a}}$ \\
\hline C-peptide $(\mathrm{nmol} / \mathrm{l})$, mean $(\mathrm{SD})^{\mathrm{b}}$ & - & $1.35(0.64)$ & $0.63(0.47)$ & $<0.0001^{\mathrm{a}}$ \\
\hline HOMA-IR, mean (SD) ${ }^{\mathrm{b}}$ & - & $4.9(5.9)$ & $5.9(19.3)$ & $0.6239^{\mathrm{a}}$ \\
\hline HOMA- $\beta$, mean $(\mathrm{SD})^{\mathrm{b}}$ & - & $69.8(36.8)$ & $35.3(31.3)$ & $<0.0001^{\mathrm{a}}$ \\
\hline GADA (U/ml), median (interquartile range) ${ }^{\mathrm{b}}$ & - & - & $206.5(46.5,250.0)$ & - \\
\hline Insulin treatment $(\%)^{\mathrm{b}}$ & - & 4.3 & 58.0 & $<0.0001^{\mathrm{a}}$ \\
\hline First-degree relatives with type 2 diabetes (\%) & 24.4 & 49.1 & 40.3 & $0.0845^{\mathrm{a}}$ \\
\hline First-degree relatives with type 1 diabetes (\%) & 1.8 & 4.6 & 13.4 & $0.0013^{\mathrm{a}}$ \\
\hline Low birthweight $(<3 \mathrm{~kg})(\%)$ & 14.1 & 22.3 & 21.6 & $0.0344^{\mathrm{c}}$ \\
\hline Birthweight (kg), mean (SD) & $3.51(0.65)$ & $3.46(0.75)$ & $3.39(0.69)$ & $0.0526^{\mathrm{c}}$ \\
\hline
\end{tabular}

${ }^{\text {a }}$ Difference between LADA and type 2 diabetes

${ }^{\mathrm{b}}$ Information is available for patients with LADA and type 2 diabetes only

${ }^{\mathrm{c}}$ Difference between LADA and controls

No., number 
Table 2 ORs for LADA and type 2 diabetes in relation to birthweight

\begin{tabular}{|c|c|c|c|c|c|c|c|}
\hline \multirow[t]{2}{*}{ Birthweight } & \multirow[t]{2}{*}{ No. of cases/controls } & \multicolumn{2}{|c|}{ Model 1} & \multicolumn{2}{|c|}{ Model 2} & \multicolumn{2}{|c|}{ Model 3} \\
\hline & & OR & $(95 \% \mathrm{CI})$ & OR & $(95 \% \mathrm{CI})$ & OR & $(95 \% \mathrm{CI})$ \\
\hline \multicolumn{8}{|l|}{ LADA } \\
\hline$\geq 4.0 \mathrm{~kg}$ & $22 / 131$ & 1 & Ref. & 1 & Ref. & 1 & Ref. \\
\hline $3.0-<4.0 \mathrm{~kg}$ & $83 / 387$ & 1.37 & $(0.81,2.31)$ & 1.39 & $(0.82,2.36)$ & 1.41 & $(0.83,2.41)$ \\
\hline$<3.0 \mathrm{~kg}$ & $29 / 85$ & 2.33 & $(1.23,4.42)$ & 2.31 & $(1.21,4.41)$ & 2.38 & $(1.23,4.60)$ \\
\hline Per kg birthweight & $134 / 603$ & 1.44 & $(1.07,1.95)$ & 1.45 & $(1.07,1.96)$ & 1.52 & $(1.12,2.08)$ \\
\hline \multicolumn{8}{|l|}{ Type 2 diabetes } \\
\hline$\geq 4.0 \mathrm{~kg}$ & $89 / 131$ & 1 & Ref. & 1 & Ref. & 1 & Ref. \\
\hline $3.0-<4.0 \mathrm{~kg}$ & $183 / 387$ & 0.77 & $(0.55,1.09)$ & 0.98 & $(0.64,1.48)$ & 0.97 & $(0.64,1.48)$ \\
\hline$<3.0 \mathrm{~kg}$ & $78 / 85$ & 1.69 & $(1.09,2.63)$ & 2.55 & $(1.49,4.37)$ & 2.37 & $(1.37,4.10)$ \\
\hline Per kg birthweight & $350 / 603$ & 1.25 & $(1.02,1.54)$ & 1.60 & $(1.24,2.06)$ & 1.58 & $(1.23,2.04)$ \\
\hline
\end{tabular}

Model 1, Adjusted for age and sex; model 2, adjusted for age, sex and BMI; model 3, adjusted for age, sex, BMI and family history of diabetes No., number; ref., reference

(Fig. 1); OR was estimated as $3.26(1.69,6.29)$ when compared with individuals with high birthweight and normal adult weight. The RERI was estimated as 1.90 , but numbers were small and confidence limits wide (95\% CI -0.16, 3.96). With regard to type 2 diabetes, the combination of low birthweight and overweight conferred a 40-fold increased risk (OR 39.93 [19.27, 82.71]) for type 2 diabetes (Fig. 2), with the RERI estimated as $25.09(2.70,47.49)$.

Clinical characteristics of patients with high vs low birthweight Comparing LADA patients with birthweight above and below $3 \mathrm{~kg}$ (electronic supplementary material [ESM] Table 1) indicated that those with low birthweight $(<3 \mathrm{~kg})$ tended to be more insulin resistant (mean HOMA-IR 9.75 vs 4.73), have better beta cell function (mean HOMA- $\beta$, 46.19 vs 31.92), lower median GADA levels (60 vs $222 \mathrm{U} / \mathrm{ml}$ ) and higher mean BMI (28.0 vs $26.6 \mathrm{~kg} / \mathrm{m}^{2}$ ); however, numbers were small and potential differences not significant. In patients with type 2 diabetes, no clear differences were seen between those with high and low birthweights.

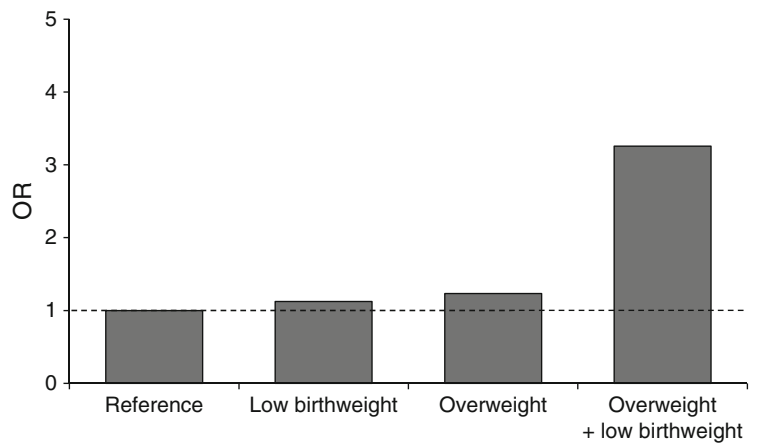

Fig. 1 ORs for LADA by combinations of birthweight and BMI. The reference is normal weight and birthweight $\geq 3 \mathrm{~kg}$

\section{Discussion}

We demonstrate that low birthweight is associated with an increased risk of type 2 diabetes [1] and we also show, for the first time, that low birthweight is linked in a similar way to the risk of LADA, with a more than twofold increased risk associated with birthweight below $3 \mathrm{~kg}$ compared with $4 \mathrm{~kg}$ or more.

The mechanisms behind the relation between birthweight and type 2 diabetes are not fully understood. According to the 'thrifty phenotype hypothesis', introduced by Hales and Barker in 1992 [2], in utero malnutrition causes a metabolic programming of thrift that, when met with rapid postnatal growth, leads to insulin resistance and type 2 diabetes [21]. It is possible that the link between LADA and low birthweight reflects the same underlying mechanism, as LADA, like type 2 diabetes, involves insulin resistance [11]. In support of this common mechanism, LADA patients with low birthweight tended to be more type-2-like, with older age at onset and indications of more pronounced insulin resistance, better beta cell function and less autoimmunity. The numbers were, however, small

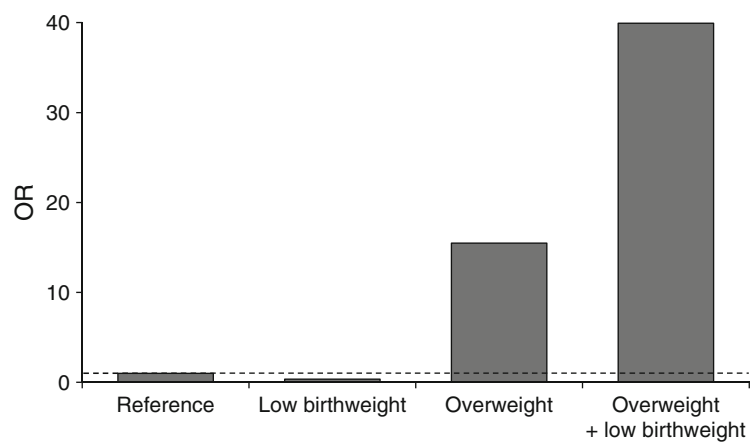

Fig. 2 ORs for type 2 diabetes by combinations of birthweight and BMI. The reference is normal weight and birthweight $\geq 3 \mathrm{~kg}$ 
and these results are only suggestive. Still, these findings are in line with previous studies $[11,22]$ that suggest that the LADA concept is heterogeneous, covering a spectrum ranging from very autoimmune, type-1-like forms to less autoimmune and more insulin-resistant forms, and risk factors may consequently differ. There was nothing to suggest that low birthweight is associated with autoimmunity per se; if anything, LADA patients with low birthweight tended to have lower GADA levels. This is in agreement with previous findings for type 1 diabetes $[9,10]$ and other autoimmune diseases, including rheumatoid arthritis [23, 24] and multiple sclerosis [25], suggesting an increased risk associated with high, but not low, birthweight.

In the present study, no information on patients with type 1 diabetes was available. However, previous findings based on all Swedish type 1 diabetes patients born in the period 19832007 [26] indicate that mean birthweight is higher in this patient group than in LADA patients $(3,540 \mathrm{~g}$ vs $3,389 \mathrm{~g}$ in our sample).

With regard to type 2 diabetes, our results were in accordance with a large number of previous studies [1]. A striking finding was the strong interaction between low birthweight and adult overweight; a 40-fold increased risk was seen in those with a combination of these risk factors. Few previous studies have investigated the influence of the combination of low birthweight and overweight in relation to diabetes, but similar findings are seen in relation to the metabolic syndrome [27] and myocardial infarction [28]. Notably, the combined effect was not as pronounced for LADA but our results did indicate that low birthweight alone is a risk factor for LADA when combined with adult overweight. These findings fit with the thrifty phenotype hypothesis [21], according to which a system permanently programmed for thrift will store body fat rather than grow muscle when exposed to an affluent nutritional intake $[29,30]$. From a public health perspective this is potentially important, as it suggests that many cases of diabetes could be prevented in this additionally predisposed group if a healthy weight is maintained.

Our results support the likelihood that environmental rather than genetic factors underlie the association between low birthweight and diabetes; adjustment for family history had limited influence. This is compatible with results from twin studies $[31,32]$. Of note, the association between low birthweight and diabetes was strengthened when individuals with diabetes in the mother were excluded. This is to be expected given that maternal hyperglycaemia may manifest in high birthweight, as well as an increased diabetes risk in the offspring [13].

Considerations of the methods The strengths of this study include a well-defined population, detailed information on a number of potential confounders and access to registry data for validation. The main limitation was the self-reported information on birthweight. The validity of such information is reportedly high $[1,7,33]$ and, in particular, we had the opportunity to validate the self-reports against registry data for the younger part of the cohort. The correlation was high $(0.86)$ and the sensitivity and specificity for correctly classifying participants with low birthweight were $90 \%$ and $100 \%$, respectively. It is possible that the quality of the self-reports was less accurate for older participants, for whom we did not have access to registry information. On the other hand, several other studies have indicated that the validity of self-reported information on birthweight is high at older ages [1, 7, 33]. In support, we saw an association between low birthweight and LADA when the analyses were restricted to older participants ( $\geq 60$ years): OR per kg reduction $1.97(1.15,3.36)$.

We could not distinguish children who were small for gestational age from preterm babies. This would have been interesting as previous studies have shown that type 2 diabetes is associated with both being small for gestational age and prematurity per se [34]. Another limitation was that only 44.6\% of the original study population provided information on birthweight - the question is whether those who did not know their birthweight differ from those included in the analysis. In this context, it is important to note that response rates were similar among cases and controls and, furthermore, that registry data indicate that mean birthweight was similar in responders and non-responders. Importantly, our findings for type 2 diabetes were in line with numerous previous studies based on registry data [1].

Notably, there is no uniform definition of LADA. Our criteria were consistent with previous reports except for the use of C-peptide, which provides a more objective measure of residual insulin secretion than lack of insulin treatment for 612 months after diagnosis, which is often used [11]. One could speculate that false-positive LADA patients, i.e. patients with type 2 diabetes erroneously identified as having LADA, may contribute to similar findings for birthweight. Still, the sensitivity of the GADA assay suggests that such misclassification is minor. Furthermore, raising the cut-off level on the assay did not change the results.

There were clear differences between the two patient groups with regard to a number of clinical characteristics. One concern is whether the birthweight of the controls reflects the birthweight of the population that generated the cases. Controls were randomly selected from the population, but a spurious association may arise if participating controls had higher birthweight than non-participating controls and consequently than the population as a whole. We have no reason to believe that birthweight would be associated with non-participation. It is noteworthy that when we compared the level of education of our controls with that of the general population in Skåne using data from Statistics Sweden, there were no differences: e.g. $32.8 \%$ vs $32.9 \%$ had a university degree. A strong argument against selection bias is the similarity of our 
findings to previous reports on type 2 diabetes based on different methods and populations [1].

Although GADA can disappear after prolonged duration of LADA [35], they tend to be more stable than in type 1 diabetes [11]. Our study was based on newly diagnosed cases, which minimises these problems. With regard to generalisability it should be noted that ethnic differences in the association between birthweight and type 2 diabetes have been suggested [1], possibly reflecting differences in the prevalence of maternal diabetes. Worth mentioning is that the proportion born outside Sweden or who were Swedish born with one or two immigrant parents was low in our sample (14.9\%) and analyses excluding these individuals did not affect the results.

Conclusions Low birthweight was associated with an increased risk of LADA as well as of type 2 diabetes. Our findings favour the role of environmental, rather than genetic, explanations for this link. This paper also supports findings from previous studies [36-39] which indicate that LADA, despite its autoimmune features, has an aetiology that, in part, may be similar to that of type 2 diabetes.

Acknowledgements Preliminary results of this work were presented as an abstract at the 50th European Association for the Study of Diabetes (EASD) Annual Meeting 2014, in Vienna, Austria.

Funding The Swedish Medical Research Council, the Swedish Research Council for Health, Working life and Welfare, AFA Insurance Company and the Swedish Diabetes Association gave grants for ESTRID. ANDIS and ANDiU were funded by a strategic grant from the Swedish Medical Research Council (Excellence of Diabetes Research in Sweden [EXODIAB]). ANDIS was also funded by ALF - the Swedish Research Council funding for Clinical Research in medicine. The funders had no influence on any aspect of this work.

Duality of interest The authors declare that there is no duality of interest associated with this manuscript.

Contribution statement LA and TT contributed to analysing and interpreting the results and critically reviewed the manuscript. SC, POC, LG, MM and PS contributed to the collection of data, interpretation of the results and writing of the manuscript. SC contributed to the design and conduct of the study. RH contributed to the conduct of the study and was responsible for analysing the data and writing the paper. $\mathrm{RH}$ and $\mathrm{SC}$ had access to all data in this study and are the guarantors of the integrity of the data and of the work as a whole. All authors critically revised and approved the final version of the manuscript

\section{References}

1. Whincup PH, Kaye SJ, Owen CG et al (2008) Birth weight and risk of type 2 diabetes: a systematic review. JAMA 300:2886-2897

2. Hales CN, Barker DJ (1992) Type 2 (non-insulin-dependent) diabetes mellitus: the thrifty phenotype hypothesis. Diabetologia 35: 595-601
3. Jimenez-Chillaron JC, Isganaitis E, Charalambous M et al (2009) Intergenerational transmission of glucose intolerance and obesity by in utero undernutrition in mice. Diabetes 58:460-468

4. Nightingale CM, Rudnicka AR, Owen CG et al (2015) Birthweight and risk markers for type 2 diabetes and cardiovascular disease in childhood: the Child Heart and Health Study in England (CHASE). Diabetologia 58:474-484

5. Jimenez-Chillaron JC, Hernandez-Valencia M, Reamer C et al (2005) Beta-cell secretory dysfunction in the pathogenesis of low birth weight-associated diabetes: a murine model. Diabetes 54:702 711

6. Portha B, Chavey A, Movassat J (2011) Early-life origins of type 2 diabetes: fetal programming of the beta-cell mass. Exp Diabetes Res 2011:105076

7. Tyrrell JS, Yaghootkar H, Freathy RM, Hattersley AT, Frayling TM (2013) Parental diabetes and birthweight in 236030 individuals in the UK Biobank study. Int J Epidemiol 42:1714-1723

8. Hattersley AT, Tooke JE (1999) The fetal insulin hypothesis: an alternative explanation of the association of low birthweight with diabetes and vascular disease. Lancet 353:1789-1792

9. Harder T, Roepke K, Diller N, Stechling Y, Dudenhausen JW, Plagemann A (2009) Birth weight, early weight gain, and subsequent risk of type 1 diabetes: systematic review and meta-analysis. Am J Epidemiol 169:1428-1436

10. Cardwell CR, Stene LC, Joner G et al (2010) Birthweight and the risk of childhood-onset type 1 diabetes: a meta-analysis of observational studies using individual patient data. Diabetologia 53:641651

11. Tuomi T, Santoro N, Caprio S, Cai M, Weng J, Groop L (2014) The many faces of diabetes: a disease with increasing heterogeneity. Lancet 383:1084-1094

12. Hawa MI, Kolb H, Schloot N et al (2013) Adult-onset autoimmune diabetes in Europe is prevalent with a broad clinical phenotype: Action LADA 7. Diabetes Care 36:908-913

13. Catalano PM (2010) The impact of gestational diabetes and maternal obesity on the mother and her offspring. J Dev Orig Health Dis $1: 208-215$

14. Lofvenborg JE, Andersson T, Carlsson PO et al (2014) Coffee consumption and the risk of latent autoimmune diabetes in adults - results from a Swedish case-control study. Diabet Med 31:799-805

15. Vandenbroucke JP, Pearce N (2012) Case-control studies: basic concepts. Int J Epidemiol 41:1480-1489

16. Rahmati K, Lernmark A, Becker C et al (2008) A comparison of serum and EDTA plasma in the measurement of glutamic acid decarboxylase autoantibodies (GADA) and autoantibodies to islet antigen-2 (IA-2A) using the RSR radioimmunoassay (RIA) and enzyme linked immunosorbent assay (ELISA) kits. Clin Lab 54: 227-235

17. Smolcic VS, Bilic-Zulle L, Fisic E (2011) Validation of methods performance for routine biochemistry analytes at Cobas 6000 analyzer series module c501. Biochem Med 21:182-184

18. The Oxford Center for Diabetes. Endocrinology \& Metabolism. Diabetes Trial Unit. HOMA Calculator. Available from www.dtu. ox.ac.uk/homacalculator, accessed 1 June 2013

19. World Health Organization (2004) International statistical classification of diseases and related health problems. Available from www.who.int/classifications/icd/ICD-10_2nd_ed_volume2.pdf, accessed 15 April 2015

20. Andersson T, Alfredsson L, Kallberg H, Zdravkovic S, Ahlbom A (2005) Calculating measures of biological interaction. Eur J Epidemiol 20:575-579

21. Hales CN, Barker DJ (2001) The thrifty phenotype hypothesis. Br Med Bull 60:5-20

22. Radtke MA, Midthjell K, Nilsen TI, Grill V (2009) Heterogeneity of patients with latent autoimmune diabetes in adults: linkage to 
autoimmunity is apparent only in those with perceived need for insulin treatment: results from the Nord-Trondelag Health (HUNT) study. Diabetes Care 32:245-250

23. Colebatch AN, Edwards CJ (2011) The influence of early life factors on the risk of developing rheumatoid arthritis. Clin Exp Immunol 163:11-16

24. Svendsen AJ, Kyvik KO, Houen G et al (2014) Newborn infant characteristics and risk of future rheumatoid arthritis: a twin-control study. Rheumatol Int 34:523-528

25. Ramagopalan SV, Herrera BM, Valdar W et al (2008) No effect of birth weight on the risk of multiple sclerosis. A population-based study. Neuroepidemiology 31:181-184

26. Persson E, Waernbaum I (2013) Estimating a marginal causal odds ratio in a case-control design: analyzing the effect of low birth weight on the risk of type 1 diabetes mellitus. Stat Med 32:2500 2512

27. Mi J, Cheng H, Zhao XY, Hou DQ, Chen FF, Zhang KL (2008) Developmental origin of metabolic syndrome: interaction of thinness at birth and overweight during adult life in Chinese population. Obes Rev 9(suppl 1):91-94

28. Rajaleid K, Janszky I, Hallqvist J (2011) Small birth size, adult overweight, and risk of acute myocardial infraction. Epidemiology 22:138-147

29. Kensara OA, Wootton SA, Phillips DI et al (2005) Fetal programming of body composition: relation between birth weight and body composition measured with dual-energy X-ray absorptiometry and anthropometric methods in older Englishmen. Am J Clin Nutr 82: 980-987

30. Brown LD (2014) Endocrine regulation of fetal skeletal muscle growth: impact on future metabolic health. J Endocrinol 221: R13-R29
31. Iliadou A, Cnattingius S, Lichtenstein P (2004) Low birthweight and type 2 diabetes: a study on 11162 Swedish twins. Int J Epidemiol 33:948-953

32. Vaag A, Poulsen P (2007) Twins in metabolic and diabetes research: what do they tell us? Curr Opin Clin Nutr Metab Care 10:591-596

33. Wodskou PM, Hundrup YA, Obel EB, Jorgensen T (2010) Validity of self-reported birthweight among middle-aged and elderly women in the Danish Nurse Cohort Study. Acta Obstet Gynecol Scand 89: 1134-1139

34. Pilgaard K, Faerch K, Carstensen B et al (2010) Low birthweight and premature birth are both associated with type 2 diabetes in a random sample of middle-aged Danes. Diabetologia 53:2526-2530

35. Sorgjerd EP, Skorpen F, Kvaloy K, Midthjell K, Grill V (2012) Time dynamics of autoantibodies are coupled to phenotypes and add to the heterogeneity of autoimmune diabetes in adults: the HUNT study, Norway. Diabetologia 55:1310-1318

36. Rasouli B, Andersson T, Carlsson PO et al (2014) Alcohol and the risk for latent autoimmune diabetes in adults: results based on Swedish ESTRID study. Eur J Endocrinol/Eur Fed Endocr Soc 171:535-543

37. Rasouli B, Ahlbom A, Andersson T et al (2013) Alcohol consumption is associated with reduced risk of Type 2 diabetes and autoimmune diabetes in adults: results from the Nord-Trondelag Health Study. Diabet Med 30:56-64

38. Carlsson S, Midthjell K, Grill V (2007) Influence of family history of diabetes on incidence and prevalence of latent autoimmune diabetes of the adult: results from the Nord-Trondelag Health Study. Diabetes Care 30:3040-3045

39. Olsson L, Ahlbom A, Grill V, Midthjell K, Carlsson S (2012) Sleep disturbances and low psychological well-being are associated with an increased risk of autoimmune diabetes in adults. Results from the Nord-Trondelag Health Study. Diabetes Res Clin Pract 98:302-311 